\section{A reply to Edgell}

\author{
ROBERT W. FRICK \\ State University of New York \\ Stony Brook, New York
}

Edgell (1995) does not understand the goals and problems of the theoretically oriented experimenter. This lack of understanding is reflected in Edgell's comment that "theoretical work does not demand that we establish any value to an exact amount" (p. 525). The typical issue faced by theoretically oriented experimenters is not the value of some variable, or the size of an effect, but instead whether or not one variable influences another.

Edgell claims that the range null hypothesis is always appropriate. This claim ignores the requisites of science. To use a new example, physicists believe that all electrons have the exact same mass. Presumably at least one physicist made a good effort to compare the mass of two electrons. The result could be represented as a confidence interval for the difference in mass. Thus, statistics takes the physicist as far as accepting a "range" null hypothesis. However, physicists go beyond this range null hypothesis, to assert and believe the point null hypothesis. They would not be satisfied with a range null hypothesis. There is no difference between the masses of two electrons that physicists would say is "small enough" not to matter - any finding of difference in mass between electrons would be very important, no matter how small the difference. My article was about the process that physicists and psychologists use to claim a point null hypothesis. There are, no doubt, good theoretical reasons for claiming that the mass of two electrons is always the same, but no physicist would make this claim without there having been a good effort to find a difference.

As Edgell and I agree, there are two different models for the probability of the null hypothesis, one in which its probability is zero and one in which its probability is greater than zero. He claims, with no explanation, that the experimenter's choice of models is arbitrary. It is not easy to say exactly when one model should be preferred over the other. However, the choice is not arbitrary. For example, there seems to be agreement that an experiment comparing two dissimilar treatments does not have a possible null hypothesis and that parapsychology experi-

Correspondence should be addressed R. W. Frick, Psychology Department, SUNY at Stony Brook, Stony Brook, NY 11794-2500 (e-mail: rfrick@psychl.psy.sunysb.edu). ments can have a possible null hypothesis (e.g., Loftus, 1991). Allowing experimenters an arbitrary choice on this matter would be inappropriate and psychology will not tolerate it. I do not know why Edgell claims that there is nothing special about zero; perhaps we have had different experiences, or perhaps I should have said that the special word is "no"; there is a nonzero probability that one variable has no effect on a second.

Edgell and I agree that (1) the good-effort criterion violates some meta-rules for methodology, (2) everyone would prefer that these meta-rules were not violated, and (3) unfortunately statistics cannot provide a "rigorous" decision procedure for accepting the point null hypothesis. My argument is that the good-effort criterion violates minor meta-rules, whereas never allowing experimenters to accept the point null hypothesis violates a more important meta-rule. Perhaps Edgell has a different ordering for the meta-rules. I proposed the metarules so that issues such as this could be settled by discussion, not fiat.

Other issues: In his first paragraph, Edgell seems to confuse taking a Bayesian approach to hypothesis testing and taking a Bayesian (i.e., subjective) approach to probabilities. My article is about conventional hypothesis testing, which traditionally is interpreted in terms of relative frequencies - although I would not make that interpretation. Edgell's claim that the null hypothesis can be true might seem puzzling. I think he means that even though the probability of the null hypothesis's being true is "zero," the null hypothesis is not logically impossible. In other words, one can say, "Suppose the null hypothesis is true," just as one can say, "Suppose there are green men living on Mars." In his last paragraph, Edgell argues that sometimes the good-effort criterion is likely to produce errors. His claim is too weak: Sometimes the goodeffort criterion will produce errors. However, a chance of error is standard operating procedure for psychology. The fact that there are errors in some situations does not mean that errors are likely in general, so Edgell's final sentence is in logical error.

\section{REFERENCES}

EDGELL, S. (1995). Commentary on "Accepting the null hypothesis." Memory \& Cognition, 23, 525.

LoFTUS, G. R. (1991). On the tyranny of hypothesis testing in the social sciences. Contemporary Psychology, 36, 102-105.

(Accepted for publication September 15, 1994.) 